\title{
Interdisciplinary Research of Network Science and Computational Intelligence
}

\author{
Wen-Bo Du ${ }^{1}$, Gang Yan ${ }^{2}$, Raymond Chiong ${ }^{3}$, and Yong-Xiang $\mathrm{Xia}^{4}$ \\ ${ }^{1}$ School of Electronic and Information Engineering, Beihang University, Beijing, 100191, China \\ Email:wenbodu@buaa.edu.cn \\ ${ }^{2}$ School of Physics Science and Engineering, Tongji University, Shanghai, 200092, China \\ Email: gyan@tongji.edu.cn \\ ${ }^{3}$ School of Electrical Engineering and Computing, The University of Newcastle, Callaghan, NSW 2308, Australia \\ Email: raymond.chiong@newcastle.edu.au \\ ${ }^{4}$ Department of Information Science and Electronic Engineering, Zhejiang University, Hangzhou, 310058, China \\ Email: xiayx@zju.edu.cn
}

\section{Preface}

Many influential algorithms and their variants in computational intelligence, such as artificial neural networks, particle swarm optimization, and genetic algorithm, mimic real complex systems composed of a large number of components which interact with each other. Network science focuses on the relationships between different components in a system and exploring how the relationships give rise to collective behaviors. Hence network science offers a novel framework to represent many computational intelligence algorithms and to possibly unveil the working mechanisms that underlie these algorithms. On the other hand, computational intelligence can be used to solve many problems in real-world complex networks, such as enhancing network robustness, improving the efficiency of network routing, maximizing the influence of a node set, and so on. Therefore, the interface between network science and computational intelligence has become the new frontier in both fields.

This special issue investigates state-of-the-art interdisciplinary research of network science and computational intelligence, focusing on:
- applying network science to analyzing and improving the performance of computational intelligence algorithms;

- applying computational intelligence to optimizing the capacity and efficiency of real network systems.

The first paper, Cascades Tolerance of Scale-Free Networks with Attack Cost, co-authored by Chen Hong, Nai-Yu Yin, Ning He, Oriol Lordan, and Jose Maria Sallan, provides an interesting study of the attack-cost-based cascading failure model and proposes an effective node attacking strategy based on the genetic algorithm (GA). Their results demonstrate that the attack cost is of significant impact on the cascade tolerance of networks and the GA based attacking strategy is more destructive than other traditional attacking strategies.

The paper A Multi-objective Evolutionary Algorithm with Dynamic Topology and Its Application on Networkwide Flight Trajectories Planning by Su Yan, Kaiquan Cai, and Majed Swaid presents an improved multi-objective evolutionary algorithm called DTNSGAII, which uses a dynamic individual interaction network topology to assist the crossover operation. Their results show that the dynamic topology can balance the needs of diversity maintenance and convergence capability of the algorithm. DTNSGAII is 
then applied to solve the flight trajectory planning problem, verifying that the application of dynamic network topology improves the performance of the NSGAII for real-world problems.

In the paper Elitism set based particle swarm optimization and its application, Yanxia Sun and Zenghui Wang propose three new PSO methods, namely PEPSO, PEPSO II, and APEPSO, in which probabilistic techniques are considered to avoid possible premature of the particles. The authors have demonstrated the good performance of these proposed new PSOs by comparing them with several commonly used PSO variants under different topologies.

One of the main challenges in network science and computational intelligence is how to efficiently manage and process the vast amount of information in graph data to support decision making. In the paper Topology Modeling and Analysis of a Power Grid Network Using a Graph Database, Bowen Kan, Wendong Zhu, Guangyi Liu, Xi Chen, Di Shi, and Weiqing Yu introduces a new technique for storing, modeling, and analyzing power grid data, based on the open source graph database Neo4j. The functionality and efficiency of this technique is demonstrated via extensive experiments and tests.

Lastly, Xiaowen Bi, Zeyu Han, and Wallace K. S. Tang present the paper entitled Evolutionary Multi-objective Optimization for Multi-depot Vehicle Routing in Logistics, which focuses on a challenging problem in logistics system, the multi-depot vehicle routing. Local-search empowered genetic operations and a fuzzy cluster-based initialization process are embedded in an advanced multi-objective evolutionary algorithm. The performance of the proposed algorithm is confirmed by extensive simulations on numerical datasets and real traffic conditions with various customers' distributions.

We wish to express our gratitude to the authors for their efforts and contributions, to the anonymous referees for their insightful and constructive reviews, and to the Editorsin-Chief for their kind help. We believe that the articles in this Special Issue will prompt the research at the interface between network science and computational intelligence. 